\title{
Inhibition of histone deacetylases by trans-cinnamic acid and its antitumor effect against colon cancer xenografts in athymic mice
}

\author{
BINGYAN ZHU, BOYANG SHANG, YI LI and YONGSU ZHEN
}

Department of Oncology, Institute of Medicinal Biotechnology, Chinese Academy of Medical Sciences and Peking Medical College, Beijing 100050, P.R. China

Received December 25, 2014; Accepted December 4, 2015

DOI: $10.3892 / \mathrm{mmr} .2016 .5041$

\begin{abstract}
Previous studies have shown that trans-cinnamic acid (tCA) has a broad spectrum of biological activities, and exhibits antioxidant, anti-inflammatory and anticancer properties. In addition, tCA and a variety of its analogs have been detected as gut microbe-derived metabolites exerting various biological effects in the colon. The aim of this study was to assess the antitumor activity of tCA in vitro and in vivo, in particular its therapeutic efficacy against colon cancer xenografts in athymic mice. Furthermore, it aimed to examine the effects of tCA on histone deacetylases (HDACs) and to identify the underlying molecular mechanisms. Using an MTT assay, tCA was observed to inhibit the proliferation of several cancer cell lines, and the half maximal inhibitory concentration (IC50) in HT29 colon carcinoma cells was $\sim 1 \mathrm{mM}$. Western blot analysis demonstrated that tCA upregulated the expression of acetyl-H3 and acetyl-H4 proteins, which was consistent with the effects of the HDAC inhibitor, trichostatin A (TSA). Furthermore, expression of Bcl-2 (a marker of cell proliferation) was reduced, and apoptosis was induced. Apoptosis was shown by the activation of cleavage of poly ADP ribose polymerase and the increased expression of Bax. Apoptosis was also confirmed using APC Annexin V and SYTOX Green Nucleic Acid Stain. In addition, the tCA-induced inhibition of the expression of HDAC markers and activation of apoptosis in tumor tissues were further confirmed by immunohistochemistry. Intragastric administration of tCA at doses of 1.0 and $1.5 \mathrm{mmol} / \mathrm{kg}$ body weight suppressed the growth of HT29 human colon carcinoma xenografts in athymic mice at well-tolerated doses. No toxic changes were found in the heart, lung, liver, kidney, colon or bone marrow following histopathological examination. This study indicated that tCA
\end{abstract}

Correspondence to: Professor Yongsu Zhen, Department of Oncology, Institute of Medicinal Biotechnology, Chinese Academy of Medical Sciences and Peking Medical College, 1 Tiantan Xili, Beijing 100050, P.R. China

E-mail: zhenysm@126.com

Key words: trans-cinnamic acid, histone deacetylase, HT29 cells, antitumor activity is effective against colon cancer xenograft in nude mice. The antitumor mechanism of tCA was mediated, at least in part, by inhibition of HDACs in cancer cells. As an endogenous microbial metabolite predominantly produced in the colon, tCA is an agent of interest for further evaluation.

\section{Introduction}

Epigenetic alteration, which refers to the regulation of gene expression via post-translational modification of the chromatin structure without changes in the underlying DNA sequence, is crucial in diverse physiological and pathological cellular processes (1). Epigenetic defects in cancer cells can be efficiently reverted by means of pharmacological inhibitors of the enzymes that are responsible for establishing/maintaining the epigenetic markers (2). In addition, disruption of the epigenome is a fundamental mechanism in cancer development, and several epigenetic drugs, including azacitidine, decitabine, vorinostat and romidepsin, have been approved for cancer treatment (3). Data derived from experiments and clinical studies clearly indicate that epigenetic drugs are effective as modifiers of cancer phenotype and positive regulators of cancer cells in cancer therapy.

Acetylation, one of the most common modifications in epigenetics, serves as a key regulatory mechanism for chromatin structure and gene expression (4). Histone deacetylases (HDACs) are a vast family of enzymes involved in chromatin remodeling and have crucial roles in numerous biological processes. The finding that overexpression of HDACs is associated with tumor initiation and progression has prompted the research and development of HDAC inhibitors (HDACIs) as anticancer agents. HDAC overexpression alters the expression and function of tumor-associated proteins that are predominantly involved in cell proliferation, migration, angiogenesis and metastasis $(5,6)$. In addition, patients suffering from cancer with aberrant HDAC expression show a positive correlation with poor prognosis $(7,8)$. HDACIs can be divided into categories of short chain fatty acids, hydroxamic acids, cyclic tetrapeptides and benzamides, the majority of which appear to act by blocking the HDAC catalytic site containing a $\mathrm{Zn}^{2+}$ ion (9). HDAC inhibition provokes accumulation of acetylated histones that become incorporated into the nucleosomes, leading to the reversal of aberrant epigenetic patterns observed in cancer cells. Evidently, epigenetic drugs represent 
a category of promising agents in cancer treatment. Given the potent antitumor effects of HDACIs, HDACs themselves may be oncogenic, which is supported by the fact that HDAC function and/or expression is perturbed in different types of cancer and is often associated with a poor prognosis (10).

In complex biological systems, small molecules often mediate microbe-microbe and microbe-host interactions. As reported, there is widespread distribution of small-molecule-encoding biosynthetic gene clusters (BGCs) in the human microbiome (11); and accordingly, a great potential for the bacterial production of drug-like molecules in humans. Data have suggested that there are hundreds of widely distributed BGCs of unknown function in the human microbiome (11). Another study showed that the chemopreventive effect of a diet rich in fiber and slowly digestible carbohydrates has been attributed, among a variety of factors, to enhanced butyrate formation in the colon (12). It also described phenylacetate and phenylbutyrate as HDACIs, which were of particular interest as these compounds could be formed from polyphenols in fruits and vegetables during intestinal passage (13). Nutrition and the microbial flora are considered to have a marked influence on the risk of developing colorectal cancer, and the formation of butyrate and other short-chain fatty acids (SCFAs) possibly exhibit a key role as chemopreventive products of microbial fermentation in the colon. Research has suggested that butyrate acts as an endogenous HDACI in the colon (14).

Cinnamic acid (CA), a constituent of Cinnamomum cassia (family, Lauraceae), exhibits a broad spectrum of biological activity including antioxidant, anti-inflammatory and anticancer activities (15-17). CA was observed to be cardioprotective in a rat model of ischemic myocardial injury and this effect was attributable to its anti-oxidative and anti-inflammatory properties, as well as the increased NO level (18). CA shows protective effects against cisplatin-induced nephrotoxicity that may be attributed to its antioxidant activities (19). CA that occurs in propolis can modulate antigen receptors, cytokine production and the fungicidal activity of human monocytes depending on its concentration (20). Studies have also suggested the possibility of using CA for preventing advanced glycation end-product-mediated diabetic complications and for diabetes management $(21,22)$. CA is a naturally occurring phenolic compound with antimicrobial activity. Cis-CA (cCA) may be a potential antimycobacterial or synergistic agent that can be developed against tuberculosis (23). As described, CA is one of the major components of Brazilian green propolis, which exhibits a gastric protective effect and anticancer activity (24). It is reported that CA may act as a skin whitening agent via the inhibition of tyrosinase activity and its expression in melanocytes (25). Furthermore, CA shows antiproliferative activity against melanoma cells (26) and lung carcinoma cells (27). In addition, a variety of CA derivatives have been reported as HDAC-binding components (28). However, the HDAC inhibitory activity of trans-CA (tCA) itself remains to be fully defined.

Although isolated from plants, tCA may also be found as an endogenous substance in human body, predominantly in the colon. It has been demonstrated that tCA is one of the intestinal metabolites of nutritional polyphenols (13). Following analysis tCA and a great variety of its closely related analogous compounds, such as, caffeic acid, coumaric acid, ferulic acid and isoferulic acid, were found to be present in feces (14). It was reported that butyrate, other SCFAs and tCA derivatives were formed during the degradation of polyphenolic constituents of fruits and vegetables by intestinal microorganisms, and inhibited global HDAC activity in nuclear extracts from HT-29 human colon carcinoma cells (14). Thus, tCA occurs as an endogenous substance in the colon and it is worthwhile to investigate its potential effects on various pathological processes, such as carcinogenesis and tumor progression in the colon.

The present study aimed to assess the effect of tCA on HDAC activity in cancer cells and the underlying molecular mechanisms; as well as to evaluate the therapeutic efficacy of tCA against colon carcinoma xenografts in athymic mice.

\section{Materials and methods}

Reagents. HT29 human colon carcinoma cells, H460 human lung carcinoma cells, A549 human lung adenocarcinoma cells, and MIA PaCa-2 human pancreatic carcinoma cells were obtained from the American Type Culture Collection (Manassas, VA, USA). Fetal bovine serum (FBS; Gibco; Thermo Fisher Scientific Inc., Waltham, MA, USA), RPMI-1640 medium (Gibco, Thermo Fisher Scientific Inc.) and Dulbecco's modified Eagle's medium (DMEM; Hyclone; Thermo Fisher Scientific Inc.) were used in the experiments. All antibodies were purchased from Cell Signaling Technology, Inc. (Danvers, MA, USA). APC Annexin V was purchased from Invitrogen (Thermo Fisher Scientific Inc.) and SYTOX Green Nucleic Acid Stain was from BD Biosciences (Franklin Lakes, NJ, USA). Fluorescein isothiocyanate (FITC)-dextran and tCA were purchased from Sigma-Aldrich (St. Louis, MO, USA). EIPA was obtained from Thermo Fisher Scientific Inc. The HDAC-GloTM I/II Assay and Screening system were obtained from Promega Corporation (Waltham, MA, USA).

Cell culture. HT29, H460 and A549 cells were grown in modified RPMI-1640 supplemented with $10 \%$ heat-inactivated FBS, penicillin $\mathrm{G}(100 \mathrm{U} / \mathrm{ml})$ and streptomycin $(100 \mu \mathrm{g} / \mathrm{ml})$ (both from North China Pharmaceutical, Co., Ltd., Shijiazhuang, China). MIA PaCa-2 cells were grown in DMEM supplemented with the same substances. All cell lines were cultured at $37^{\circ} \mathrm{C}$ in a humidified $5 \% \mathrm{CO}_{2}$ incubator.

Cytotoxicity assay. Cells were plated in 96-well plates at a density of 3,000 cells per well and incubated for $24 \mathrm{~h}$ at $37^{\circ} \mathrm{C}$. Then, different concentrations $(0.09,0.17,034,0.68,1.36$ and $2.72 \mathrm{mM}$ ) of tCA were added. At $48 \mathrm{~h}$, MTT (Amresco Inc, Solon, OH, USA) was added to each well and further incubated for another $4 \mathrm{~h}$. The medium containing MTT solution was then discarded, and $150 \mu \mathrm{l}$ dimethyl sulfoxide was added to each well. The absorbance (A) at $570 \mathrm{~nm}$ was detected with a microplate reader (Multiskan MK3; Thermo Fisher Scientific, Inc.). Untreated cells served as a control. The tCA relative cell viability compared with the control group was calculated according to the following formula: Cell viability $(\%)=\left[\left(\mathrm{A}_{\text {treat }}\right.\right.$ $\left.-\mathrm{A}_{\text {blank }}\right)$ / $\left.\left(\mathrm{A}_{\text {control }}-\mathrm{A}_{\text {blank }}\right)\right]$ x 100.

HDAC activity assay in a cell-free system and in cultured cells. HDAC activity in the cell-free system and in cultured cells 
was detected with the HDAC-GloTM I/II Assay and Screening system following the standard protocol (29). A HDAC assay was performed in a white 96-well plate. For the HDAC activity assay in the cell-free system, cell lysate was incubated with tCA and trichostatin A (TSA) in HDAC assay buffer $(100 \mathrm{ml})$ at $37^{\circ} \mathrm{C}$ for $60 \mathrm{~min}$ and then $100 \mathrm{ml} \mathrm{HDACTM} \mathrm{I/II} \mathrm{reagent}$ were added. After $30 \mathrm{~min}$, luminescence was measured. For the HDAC assay in cultured cells, 10,000 cells per well were treated with tCA or control agents for 6 or $12 \mathrm{~h}$ (for tCA and TSA in Hela nuclear extract, the concentrations were 6.25, 12.5, 25, 50, 100 and $200 \mu \mathrm{M}$; for tCA in HT29 cells, 187.5, 375.5, $750,1,500$ and 3,000 $\mu \mathrm{M}$; and for TSA in HT29 cells, 3.125, $6.25,12.5,25$ and $50 \mathrm{nM})$. Then, cell medium was changed to serum-free medium with HDAC buffer $(50+50 \mathrm{ml})$. After $15 \mathrm{~min}$ incubation, HDAC I/II reagents $(100 \mathrm{ml})$ were added and luminescence was measured using Enspire 2300 multilabel reader (Perkin Elmer, Waltham, MA, USA) after $30 \mathrm{~min}$.

Western blot analysis. After incubation at $37^{\circ} \mathrm{C}$ for $12 \mathrm{~h}$, ice-cold phosphate-buffered saline (PBS) solution was used twice to rinse cells. Then cells were lysed with cell lysis buffer and the dishes were incubated for $10-30 \mathrm{~min}$ at $4^{\circ} \mathrm{C}$. Cells were scraped into lysis buffer, and the lysates were clarified by centrifugation at $15,294 \mathrm{x}$ g for $15 \mathrm{~min}$ at $4^{\circ} \mathrm{C}$. Protein concentrations were determined using a Bicinchoninic acid Protein Assay kit (Bio-Rad, Hercules, CA, USA) and western blotting was performed. Briefly, an equal amount of total protein extracted from cultured cells was separated by $12 \%$ sodium dodecyl sulfate-polyacrylamide gel electrophoresis and transferred to polyvinylidene difluoride (PVDF; Millipore, Billerica, MA, USA) membranes. The following primary antibodies and horseradish peroxidase-conjugated appropriate secondary antibodies were used to detect the designated proteins: Acetyl-histone H3 (Ac-H3), (Lys9) rabbit monoclonal antibody $(1: 1,000$; cat. no. \#9649; Cell Signaling Technology Inc.); Ac-H4 (Lys8) rabbit polyclonal antibody (1:1,000; cat. no. \#2594; Cell Signaling Technology Inc.); $\beta$-actin rabbit polyclonal antibody $(1: 5,000$; cat. no. SC-1616, Santa Cruz Biotechnology Inc., Santa Cruz, CA, USA); PARP polyclonal antibody (1:1,000; cat. no. \#9542, Cell Signaling Technology Inc.); Bax rabbit monoclonal antibody (1:1,000; cat. no. \#5023, Cell Signaling Technology Inc.); Bcl-2 rabbit monoclonal antibody (1:1,000; cat. no. \#2870, Cell Signaling Technology Inc.); goat anti-mouse peroxidase-coupled antibody (cat. no. ZB-2305) and goat anti-rabbit peroxidase-coupled antibody (cat. no. ZB-2301) (1:5,000; Zhongshan Goldenbridge Biotechnology Co., Ltd., Beijing, China). The bound secondary antibodies on the PVDF membrane combined with enhanced chemiluminescence detection reagents (Pierce Biotechnology, Waltham, MA, USA) and exposed to X-ray films (Kodak, Tokyo, Japan), according to the manufacturer's protocol.

Cell apoptosis assay. The cell apoptosis assay was performed using APC Annexin V and SYTOX Green Nucleic Acid staining. In brief, cultured HT29 cells were washed with cold PBS and combined with binding buffer. Cells were then incubated with APC Annexin V for 10 min and SYTOX Green Nucleic Acid Stain for another $10 \mathrm{~min}$ at $4^{\circ} \mathrm{C}$ in the dark. Then, the stained cells were observed and images were captured with confocal microscopy LSM 710 (Zeiss, Weimar, Germany) within $60 \mathrm{~min}$.

Therapeutic experiment with HT29 colon carcinoma xenografts. Female BALB/c nude mice (age, 4-6 weeks) were purchased from the Institute for Experimental Animals, Chinese Academy of Medical Sciences \& Peking Union Medical College (Beijing, China) and allowed to adapt to study environment for one week prior to the experiment. The study protocols were in accordance with the regulations of Good Laboratory Practice for non-clinical laboratory studies of drugs issued by the National Scientific and Technologic Committee of People's Republic of China. This study was approved by the animal care committee of the Institute of Medicinal Biotechnology, Chinese Academy of Medical Sciences and Peking Union Medical College (Beijing, China). HT29 colon carcinoma cells $\left(1 \times 10^{7}\right)$ suspended in $200 \mu \mathrm{l}$ sterile saline were inoculated subcutaneously into the right armpit of nude mice. After $\sim 3$ weeks, tumors in the donor animals were aseptically dissected and cut into $2-\mathrm{mm}^{3}$ blocks. Then, the tumor tissue was transplanted subcutaneously by a trocar into nude mice. When the tumor size reached $\sim 100 \mathrm{~mm}^{3}$, mice were divided into the following groups $(n=6)$ : Treatment groups with different doses of tCA (1.0 and $1.5 \mathrm{mmol} / \mathrm{kg}$ respectively) and the control group. tCA was intragastrically administered, 3 times a week for a total of 6 doses. During the experiment, the long diameter and the perpendicular short diameter of HT29 xenografts were measured every 2 days for volume calculation and statistical analysis. Tumor volume was estimated by the following formula: $\mathrm{V}=0.5 \mathrm{axb}^{2}$, where $\mathrm{a}$ and $\mathrm{b}$ represented the long and the perpendicular short diameters of the tumor, respectively. Thirty-five days after inoculation, the animals were sacrificed by overdose of isoflurane anesthesia (Shandong Keyuan Pharmaceutical Co., Ltd., Jinan, China) and the xenograft tumors were removed and weighed. Specimens taken from various organs including the heart, lung, liver, pancreas, small intestine, large intestine, kidney, spleen and bone marrow of the femur were preserved in a $4 \%$ formaldehyde solution for further evaluation.

Immunohistochemistry. HT29 xenograft specimens were used for immunohistochemical detection of related proteins, such as acetyl-H3, cleaved PARP and cleaved caspase-3. Sections $(4 \mu \mathrm{m})$ were deparaffinized and rehydrated with xylene and graded alcohol solutions. After washing with PBS, endogenous peroxidase activity was quenched with $3 \%$ hydrogen peroxide. Sections were boiled in $10 \mathrm{mM}$ citrate buffer (pH 6.0; Zhongshan Goldenbridge Biotechnology Co., Ltd.) for $3 \mathrm{~min}$ in an autoclave sterilizer (Zealway Instrument Inc., Xiameng, China) followed by cooling at room temperature for $>20 \mathrm{~min}$. After rinsing with PBS, sections were incubated with all primary antibodies used in the western blot analysis (1:100 diluted in antibody diluent, Zhongshan Goldenbridge Biotechnology Co., Ltd.) for $18 \mathrm{~h}$ at $4^{\circ} \mathrm{C}$. Sections were stained with the related antibodies. After rinsing with PBS, the sections were incubated with PV6001 or PV6002 (Zhongshan Goldbridge Biotechnology Co., Ltd.) for $30 \mathrm{~min}$ at $37^{\circ} \mathrm{C}$ and stained with DAB (AR1022, Boster Biological Technology, Ltd., Wuhan, China) for 1 to $2 \mathrm{~min}$. The slides 
were counterstained with hematoxylin (Sigma-Aldrich), dehydrated with ethanol, cleared with xylene and mounted in neutral gum. Control sections were incubated with PBS instead of a primary antibody. All slides were analyzed by two independent observers.

Histopathological observation of various organs from $t C A$-treated xenograft-bearing mice. At the end of experiment, specimens were obtained from the heart, lung, liver, pancreas, small intestine, large intestine, kidney, spleen and bone marrow of the femur, of the xenograft-bearing nude mice. Histological sections $(5 \mu \mathrm{m})$ were stained with hematoxylin and eosin and observed under a light microscope (Olympus IX81, Olympus, Tokyo, Japan).

Statistical analysis. All values are expressed as the mean \pm standard deviation. Statistical analysis was conducted using one-way analysis of variance with SPSS statistical software 17.0 (SPSS Inc., Chicago, IL, USA). P<0.05 was considered to indicate a statistically significant difference.

\section{Results}

Inhibition of cancer cell proliferation by $t C A$. An MTT assay demonstrated that tCA inhibited the proliferation of HT29, MIA PaCa-2, H460 and A549 cells in a concentration-dependent manner. The IC50 values for HT29, MIA PaCa-2, H460 and A549 cells were $1.07 \pm 0.38,1.33 \pm 0.07,2.10 \pm 0.43$ and $3.54 \pm 0.34 \mathrm{mM}$, respectively. Among the tested cell lines, HT29 cells were the most sensitive to tCA.

Increase of histone acetylation in cultured cancer cells and in vivo. The present study determined whether tCA treatment resulted in increased acetylation of histone proteins in HT29 cells and MIA PaCa-2 cells. HDACI/II activity was detected for Hela nuclear extract and HT29 cells treated with various doses of tCA and TSA. As shown, tCA directly inhibited HDAC activity similar to TSA. Western blotting of the extracted protein from HT29 cells and MIA PaCa-2 cells treated with $0.4,0.8$ and $1.6 \mathrm{mM}$ tCA showed a marked increase in acetyl-H3 and acetyl-H4, compared with untreated controls. The increase of acetylation observed in histone $\mathrm{H} 3$ and $\mathrm{H} 4$ after tCA exposure was similar to that observed after treatment with TSA (Fig. 1).

Induction of apoptosis by tCA in vivo and in vitro. Inhibition of HDAC activity has been shown to lead to the induction of apoptosis in cancer cells (30). The effect of tCA on apoptosis was evaluated in HT29 cells and MIA PaCa- 2 cells. The effect of tCA on the expression of apoptosis-related proteins showed that the expression level of Bax was upregulated; while the expression levels of PARP and $\mathrm{Bcl}-2$ were downregulated. tCA resulted in an increase in the expression of apoptosis-related protein Bax and a decrease in the expression levels of PARP and Bcl-2. This change in expression levels is similar to that induced by the HDAC inhibitor TSA (Fig. 2A and B). The cells undergoing apoptosis were observed by APC Annexin V and SYTOX Green Nucleic Acid staining. A significant increase in APC Annexin V and SYTOX Green Nucleic Acid stained cells was observed after tCA treatment in MIA PaCa2 cells (Fig. 2C).
Therapeutic efficacy of tCA against HT29 colon carcinoma xenograft. Based on the above experiments which demonstrated that tCA was active in the inhibition of HDAC, the suppression of cell proliferation and the induction of apoptosis, the study was extended to evaluate the therapeutic efficacy of tCA using a HT29 colon carcinoma xenograft model. As shown in Fig. 3, determined by tumor volume, tCA at doses 1.0 and $1.5 \mathrm{mmol} / \mathrm{kg}$ inhibited xenograft growth by 39 $(\mathrm{P}<0.05)$ and $52.9 \%(\mathrm{P}<0.01)$, respectively, at the termination of the experiment. The inhibition rate of tumor growth was calculated with the formula: Inhibition rate $(\%)=[1$-tumor volume (treated)/tumor volume (control)] x 100. The average daily intake of food did not differ between the control and treated groups. Moreover, body weights were not significantly different between the treated and the control animals for the duration of the study (Fig. 3B), suggesting that the administered doses of tCA were well-tolerated.

Immunohistochemical examination on HT29 tumor tissue. To determine the location of related proteins, immunohistochemical analysis was performed using HT29 cancer xenograft tissues from the control and tCA-treated nude mice. Using immunohistochemical staining, it was demonstrated that the expression of Ac-H3 in tCA-treated HT29 cancer xenograft tissues was higher than that of control (Fig. 4A). Ac-H3 and cleaved caspase 3 were overexpressed in tCA-treated HT29 cancer tissues compared with the control tissue. Cleaved PARP was also overexpressed in tCA-treated HT29 cancer tissues compared with the control tissue. In addition, the expression of Bax increased and that of Bcl-2 decreased following tCA treatment (Fig. 4).

Histopathological observation of various organs from $t C A$-treated xenograft-bearing mice. At the end of experiment, specimens were obtained from the heart, lung, liver, pancreas, small intestine, large intestine, kidney, spleen and bone marrow of the femur, of the xenograft-bearing nude mice. No toxicopathological changes were identified in any of the tested organs (Fig. 5).

\section{Discussion}

Epigenetic programs are now widely recognized as being critical to the biological processes of cancer genesis. It has been recognized in recent years that HDACs are promising targets for therapeutic interventions intended to reverse aberrant acetylation states. In the present study, it was shown by western blotting that tCA upregulated the expression of acetyl-H3 and acetyl-H4 proteins in a dose-dependent manner in treated cells and in the tumor tissue of treated mice, which was consistent with the well-known HDAC inhibitor TSA. Therefore, tCA could be confirmed as an HDAC inhibitor. Furthermore, it was shown that tCA inhibited the proliferation of HT29, H460, A549 and MIA PaCa-2 cell lines, and the IC50 value for HT29 colon carcinoma cells was $\sim 1 \mathrm{mM}$. According to a previous study determined by another viability assay, the IC50 value for HT-144 cells was $2.4 \mathrm{mM}$; and activated-caspase 3 staining demonstrated the induction of apoptosis in HT-144 cells $24 \mathrm{~h}$ after exposure to $3.2 \mathrm{mM} \mathrm{CA}$ (26). As compared with that of classical inhibitors, such as TSA, the activity of tCA is modest. 
A

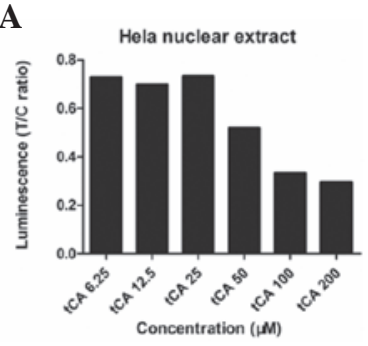

E

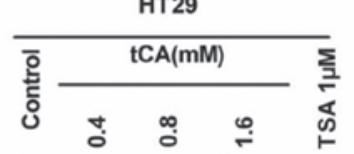

Ac-H3

Ac- 44

$\beta$ - actin

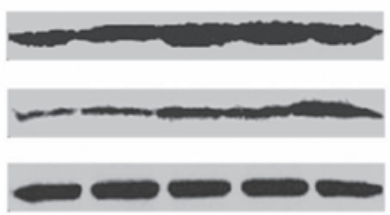

H

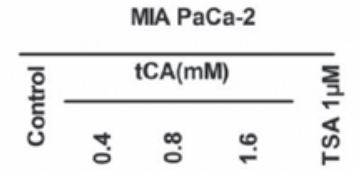

Ac- $-\mathrm{H} 3$

Ac-H4

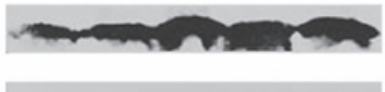

$\beta$ - actin

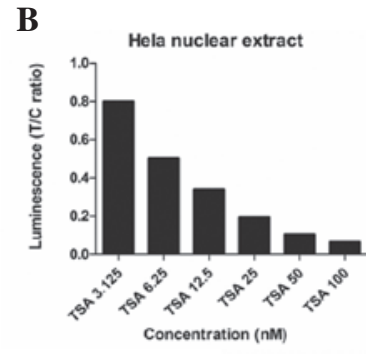

F

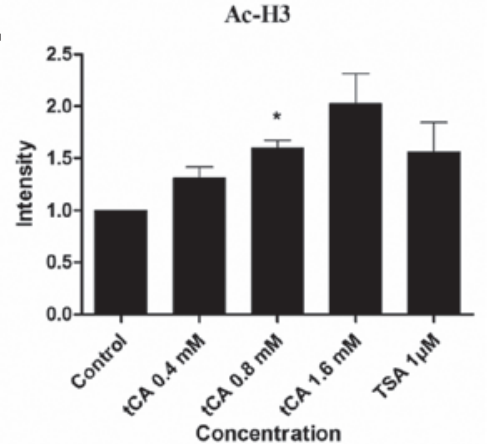

Ac-H3

I

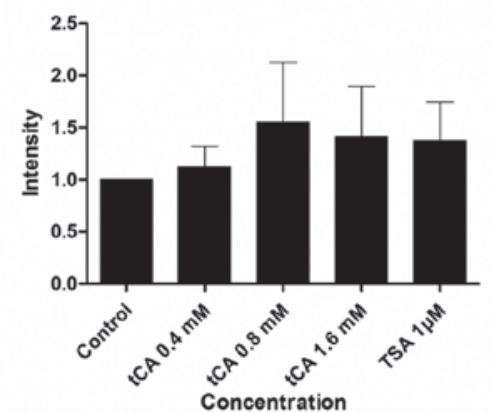

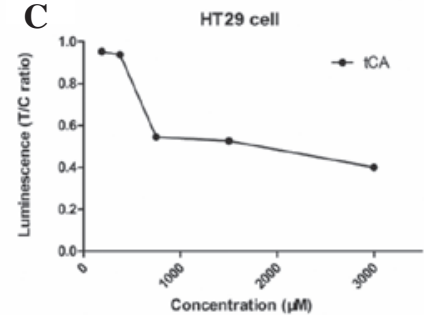

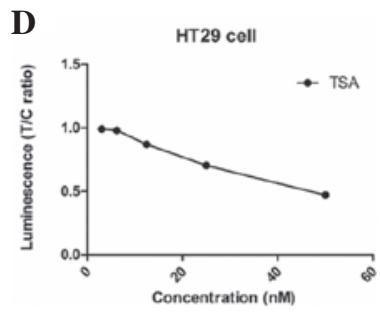

Ac-H4

G

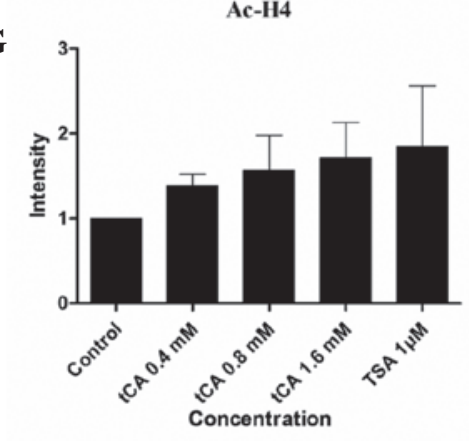

Ac-H4

J

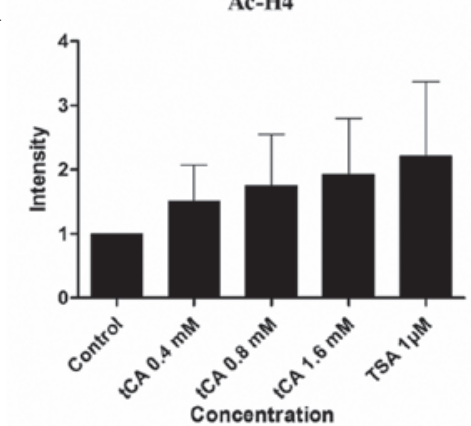

Figure 1. Effects of tCA and TSA on HDAC activity and the expression of related proteins. Hela nuclear extract was treated with various doses of (A) tCA and (B) TSA for $1 \mathrm{~h}$ and HDACI/II activity was detected. (C) tCA and (D) TSA inhibited HDAC activity in cultured cells. HT29 cells were treated with various doses of tCA for either 6 or $12 \mathrm{~h}$ and HDACI/II activity was detected. (E) Effect of tCA on histone H3 and H4 acetylation in HT29 cells as determined by western blot analysis. Quantification of (F) Ac-H3 and (G) Ac-H4 expression. (H) Effect of tCA on histone H3 and H4 acetylation in MIA PaCa-2 cells as determined by western blot analysis. Quantification of (I) Ac-H3 and (J) Ac-H4 expression. The HDAC inhibitor TSA was used as a positive control. * $\mathrm{P}<0.05$ vs. control. tCA, trans-cinnamic acid; HDAC, histone deacetylases; AC, acetyl; TSA, trichostatin A.

For the inhibition of cancer cell proliferation, the concentration of tCA reaching $\mathrm{mM}$ levels was required; however, the study data verified that tCA interfered in histone acetylation via inhibiting HDAC, leading to cancer cell cytotoxicity. In addition, the therapeutic efficacy of tCA has been confirmed. Due to its low toxicity, tCA could suppress the growth of colon carcinoma HT29 xenografts at well-tolerated doses. With regards to the mechanism of action, it is possible that tCA and HDAC were in a binding state under physiological conditions; however, an in depth study is required to determine the precise mechanism of action and the specificity of tCA binding to HDAC.

In recent years, the interaction between commensal microorganisms and the host in the development of diseases has caused much concern. The intricate relationships between microbiota and host may benefit the host in a number of ways; however, may also carry risks for disease development. One of the most important topics is whether these microorganisms can produce bioactive substances that are beneficial to health, in particular, effective for the treatment of diseases.
A recent report showed that lactocillin, a thiopeptide antibiotic produced by a member of the vaginal microbiota, had potent antibacterial activity against a range of Gram-positive vaginal pathogens (11). As reported, there was a widespread distribution of small-molecule-encoding BGCs in the human microbiome; accordingly, a great potential for the bacterial production of drug-like molecules in humans. Data further highlighted the fact that there are hundreds of widely distributed BGCs of unknown function in the human microbiome (11). In total $99 \%$ of the microbial mass is located in the gastrointestinal tract, with the majority in the colon. Therefore, attention should be paid to those active substances derived from the microbes existing in the colon. A study showed that the chemopreventive effect of a diet rich in fiber and slowly digestible carbohydrates had been attributed, among a variety of factors, to enhanced butyrate formation in the colon (12). In addition, phenylacetate and phenylbutyrate, which are active HDAC inhibitors, are formed from polyphenols in fruits and vegetables during intestinal passage (13). Thus, nutrition and the microbial flora are considered to 
A
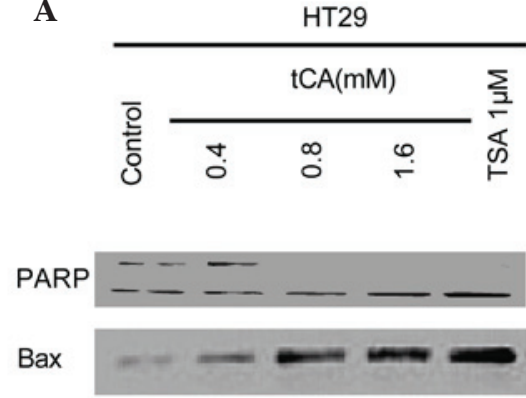

$\mathrm{Bcl}-2$

$\beta$-actin

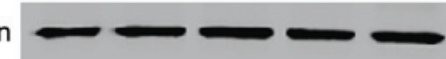

B

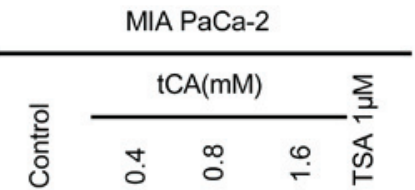

PARP



$\beta$-actin
C

A1

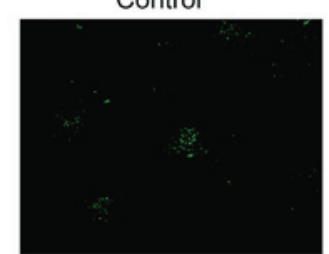

B1

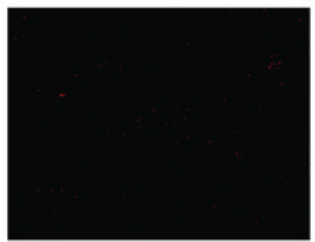

C1

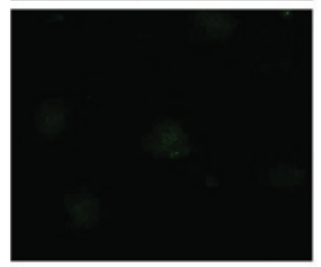

tCA $0.4 \mathrm{mM}$
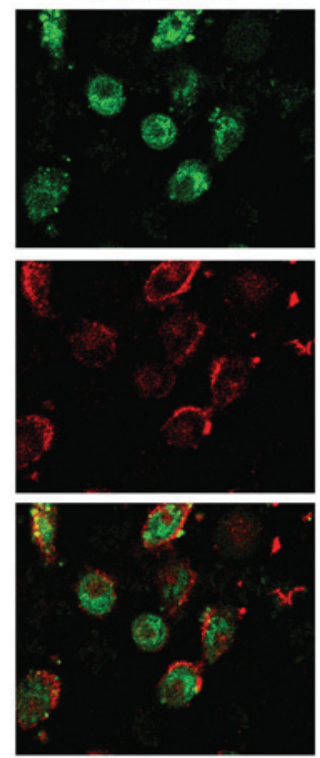

tCA $0.8 \mathrm{mM}$
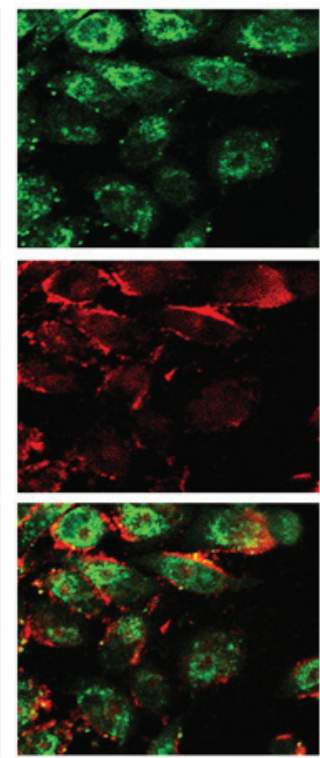

Figure 2. Effect of tCA on apoptosis of HT29 cells. Western blot analysis of PARP, Bax and Bcl-2 in (A) HT29 and (B) MIA PaCa-2 cells following treatment with the doses of $0.4,0.8$ and $1.6 \mathrm{mM} \mathrm{tCA}$, similar to that of the positive control TSA. Data are presented as the mean \pm standard deviation (n=3). (C) MIA $\mathrm{PaCa}-2$ cells treated with tCA $(0.4$ and $0.8 \mathrm{mM})$ for $12 \mathrm{~h}$ underwent apoptosis as determined by APC Annexin V and SYTOX green nucleic acid staining (original magnification, $\mathrm{x} 400$ ). Annexin $\mathrm{V}$ can bind to phosphatidylserine and SYTOX Green nucleic acid stain is impermeable to live cells. The combination can be used to identify apoptotic cells in which membrane asymmetry is lost and phosphatidylserine is translocated to the external leaflet. A1, SYTOX green staining; B1, APC Annexin V staining; C1, merge. tCA, tran-cinnamic acid; PARP, poly ADP ribose polymerase.
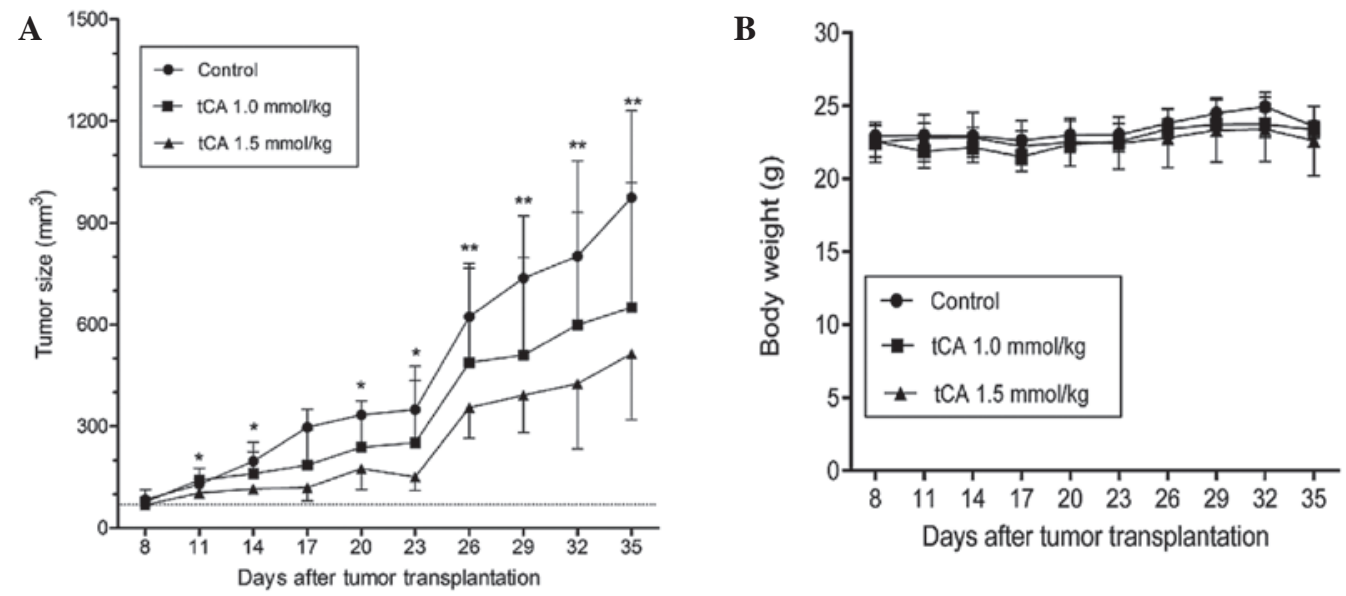

Figure 3. Antitumor efficacy of tCA against HT29 human colon carcinoma xenografts in nude mice. (A) Growth curves of HT29 xenografts in different groups ( $\mathrm{n}=6$ per group) are shown. tCA treatment suppressed the growth of tumors compared with the control. ${ }^{*} \mathrm{P}<0.05$ and ${ }^{* *} \mathrm{P}<0.01$, compared with control. (B) Body weight curves for the treated and the control animals. Values are presented as the mean \pm standard deviation. tCA, trans-cinnamic acid. 
Control

A

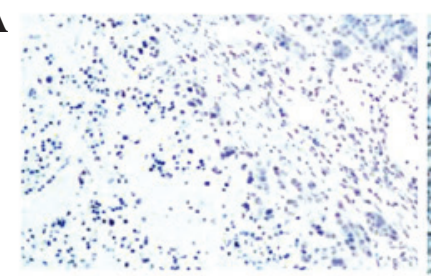

C

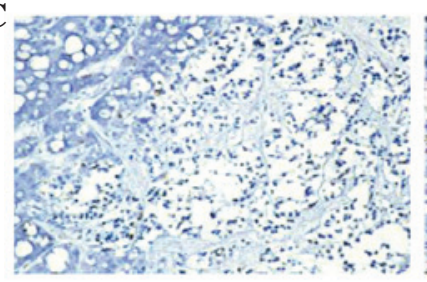

tCA $1.5 \mathrm{mmol} / \mathrm{kg}$
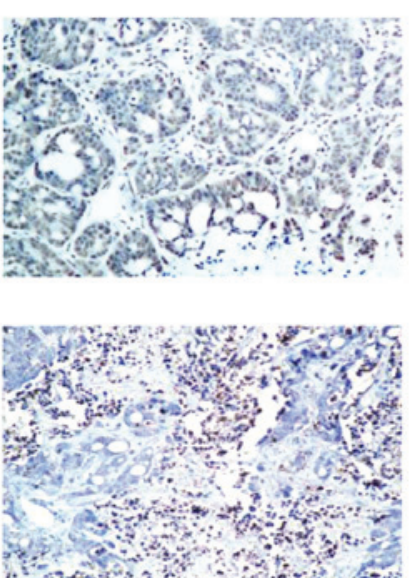

Control

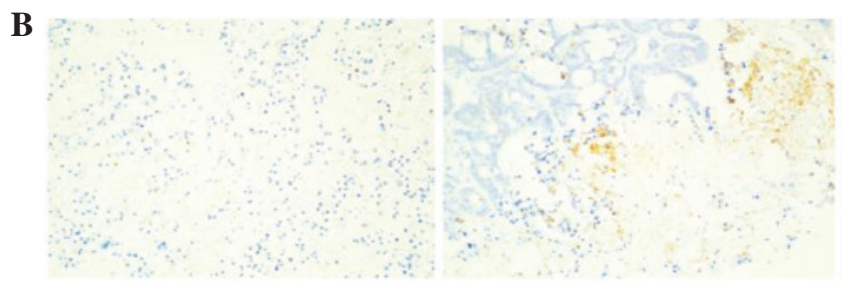

D

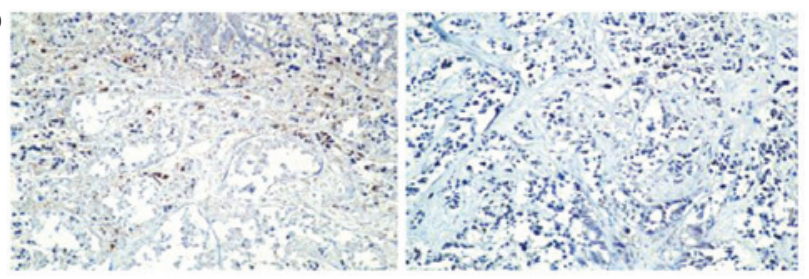

$\mathbf{E}$

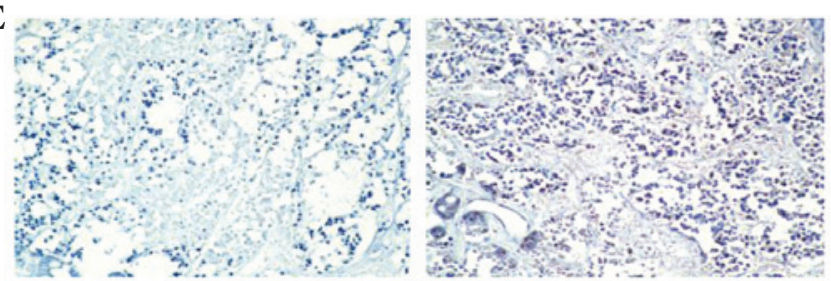

Figure 4. Immunohistochemistry staining of HT29 tumors in nude mice from the tCA-treated group and the control group. Original magnification, x100. (A) Acetyl-H3; (B) cleaved caspase 3; (C) cleaved poly ADP ribose polymerase; (D) Bcl-2; and (E) Bax. tCA, trans-cinnamic acid.

Control

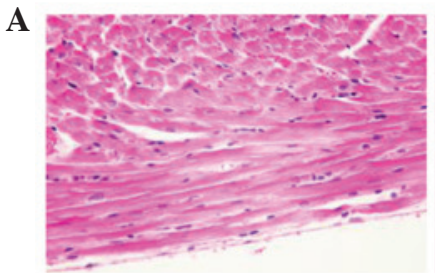

C

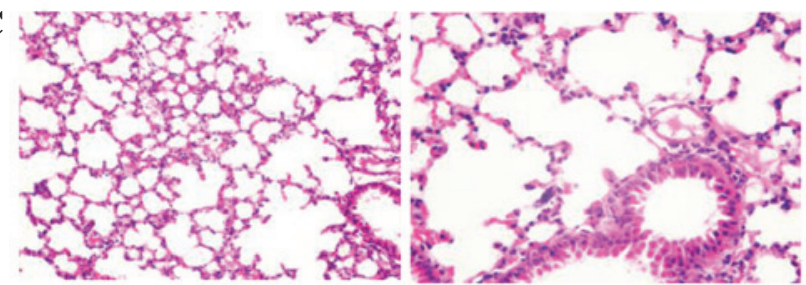

$\mathbf{E}$

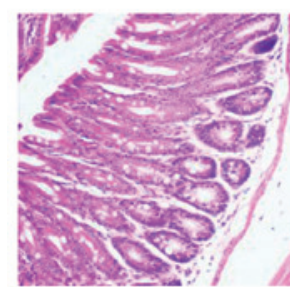

tCA1.5 mmol/kg
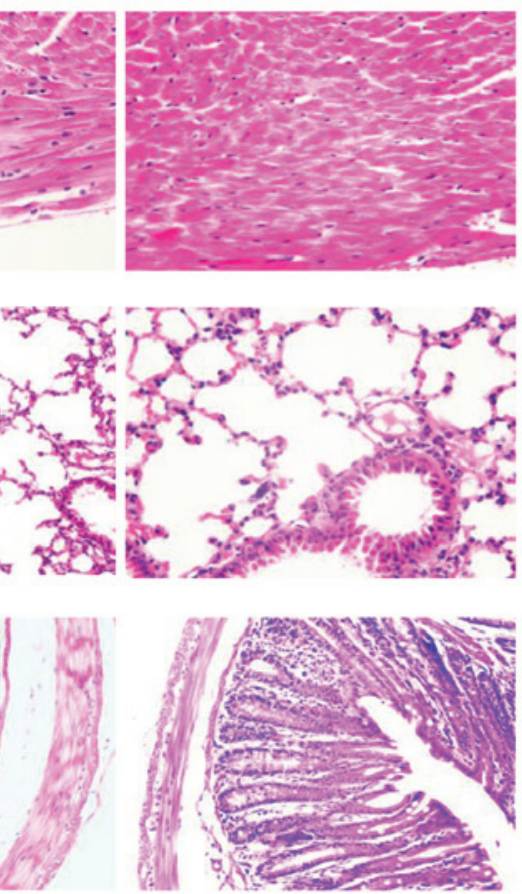

Control
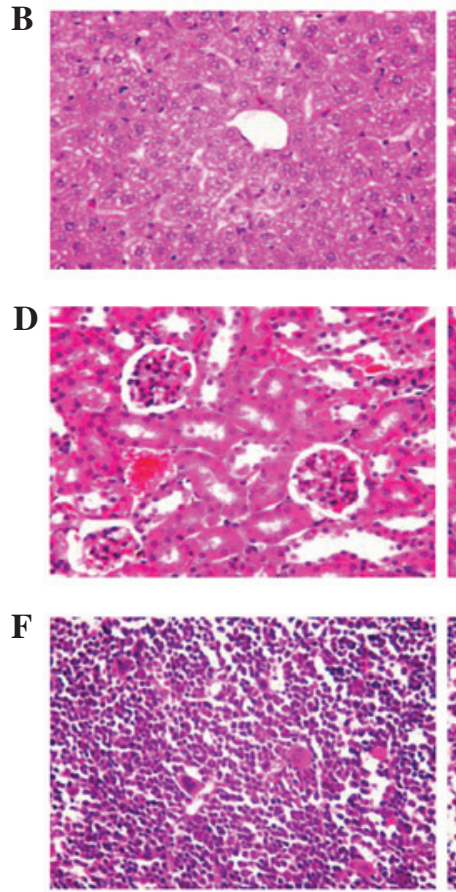

tCA1.5 mmol/kg
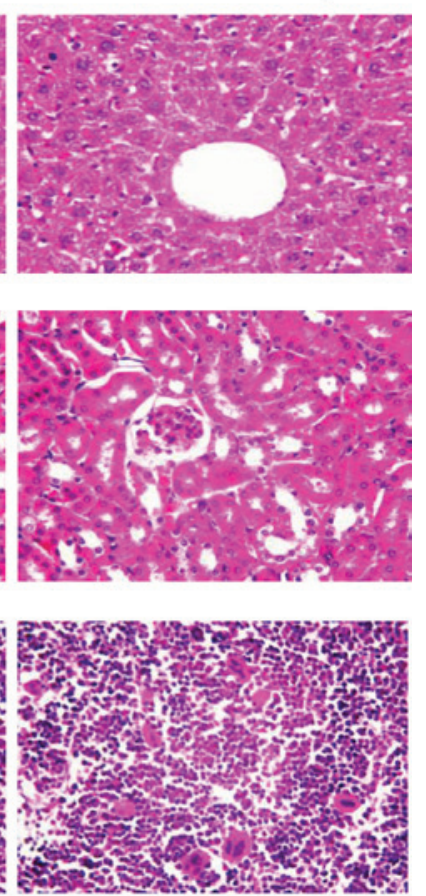

Figure 5. Histological appearance of various organs of tCA-treated (1.5 mmol/kg) and control xenograft-bearing mice. (A) heart; (B) liver; (C) lung; (D) kidney; (E) large intestine; (F) bone marrow of the femur. No toxicopathological changes were found in all of the tested organs. Stain, hematoxylin and eosin; magnification $\mathrm{x} 200$ for $\mathrm{E}$, and $\mathrm{x} 400$ for all other tissues. tCA, trans-cinnamic acid.

have a marked influence on the risk of colorectal cancer, as the formation of butyrate and other short-chain fatty acids produced by microbial fermentation possibly exhibit a chemopreventive role. A previous study demonstrated that butyrate exhibited a role as an endogenous HDAC inhibitor in the colon (14). The present study demonstrated that tCA 
as a HDAC inhibiting agent exhibits therapeutic efficacy against colon carcinoma xenografts, implying the potential of developing cancer-active compounds derived from microorganisms that reside in the intestinal tract, particularly in the colon.

In conclusion, this study indicates that tCA is effective against colon cancer xenograft in nude mice. The antitumor mechanism of tCA could be mediated, at least in part, by inhibiting HDAC in cancer cells. Considering the fact that tCA can be produced by the metabolic activity of the microorganisms existing in the intestinal tract, the antitumor efficacy of tCA warrants further investigation.

\section{Acknowledgements}

This study was supported by 'Significant New Drug Development' Major Science and Technology Projects of China (grant nos. 2013ZX09102064 and 2012ZX09301002-001-015).

\section{References}

1. Jaenisch R and Bird A: Epigenetic regulation of gene expression: How the genome integrates intrinsic and environmental signals. Nat Genet (33 Suppl): 245-254, 2003.

2. Yoo CB and Jones PA: Epigenetic therapy of cancer: Past, present and future. Nat Rev Drug Discov 5: 37-50, 2006.

3. Knower KC, To SQ, Leung YK, Ho SM and Clyne CD. Endocrine disruption of the epigenome: a breast cancer link. Endocr Relat Cancer 21: T33-T55, 2014.

4. Sterner DE and Berger SL: Acetylation of histones and transcription-related factors. Microbiol Mol Biol Rev 64: 435-459, 2000

5. Glozak MA and Seto E: Histone deacetylases and cancer. Oncogene 26: 5420-5432, 2007.

6. Xu WS, Parmigiani RB and Marks PA: Histone deacetylase inhibitors: Molecular mechanisms of action. Oncogene 26 : 5541-5552, 2007.

7. Mitsiades CS, Mitsiades NS, McMullan CJ, Poulaki V, Shringarpure R, Hideshima T, Akiyama M, Chauhan D, Munshi N, $\mathrm{Gu} \mathrm{X}$, et al: Transcriptional signature of histone deacetylase inhibition in multiple myeloma: Biological and clinical implications. Proc Natl Acad Sci USA 101: 540-545, 2004.

8. Zhang Z, Yamashita H, Toyama T, Sugiura H, Omoto Y, Ando Y, Mita K, Hamaguchi M, Hayashi S and Iwase H: HDAC6 expression is correlated with better survival in breast cancer. Clin Cancer Res 10: 6962-6968, 2004.

9. Vannini A, Volpari C, Filocamo G, Casavola EC, Brunetti M, Renzoni D, Chakravarty P, Paolini C, De Francesco R, Gallinari P, et al: Crystal structure of a eukaryotic zinc-dependent histone deacetylase, human HDAC8, complexed with a hydroxamic acid inhibitor. Proc Natl Acad Sci USA 101: 15064-15069, 2004.

10. Falkenberg KJ and Johnstone RW: Histone deacetylases and their inhibitors in cancer, neurological diseases and immune disorders. Nat Rev Drug Discov 13: 673-691, 2014.

11. Donia MS, Cimermancic P, Schulze CJ, Wieland Brown LC, Martin J, Mitreva M, Clardy J, Linington RG and Fischbach MA: A systematic analysis of biosynthetic gene clusters in the human microbiome reveals a common family of antibiotics. Cell 158: 1402-1414, 2014

12. Marks PA, Richon VM, Miller T and Kelly WK: Histone deacetylase inhibitors. Adv Cancer Res 91: 137-168, 2004.
13. Jenner AM, Rafter J and Halliwell B: Human fecal water content of phenolics: The extent of colonic exposure to aromatic compounds. Free Radic Biol Med 38: 763-772, 2005.

14. Waldecker M, Kautenburger T, Daumann H, Busch C and Schrenk D: Inhibition of histone-deacetylase activity by short-chain fatty acids and some polyphenol metabolites formed in the colon. J Nutr Biochem 19: 587-593, 2008.

15. Zhang LP and Ji ZZ: Synthesis, antiinflammatory and anticancer activity of cinnamic acids, their derivatives and analogues. Yao Xue Xue Bao 27: 817-823, 1992.

16. Akao Y, Maruyama H, Matsumoto K, Ohguchi K, Nishizawa K, Sakamoto T, Araki Y, Mishima S and Nozawa Y: Cell growth inhibitory effect of cinnamic acid derivatives from propolis on human tumor cell lines. Biol Pharm Bull 26: 1057-1059, 2003.

17. Foti MC, Daquino $C$ and Geraci C: Electron-transfer reaction of cinnamic acids and their methyl esters with the $\mathrm{DPPH}\left({ }^{*}\right)$ radical in alcoholic solutions. J Org Chem 69: 2309-2314, 2004.

18. Song F, Li H, Sun J and Wang S: Protective effects of cinnamic acid and cinnamic aldehyde on isoproterenol-induced acute myocardial ischemia in rats. J Ethnopharmacol 150: 125-130, 2013.

19. El-Sayed el SM, Abd El-Raouf OM, Fawzy HM and Manie MF: Comparative study of the possible protective effects of cinnamic acid and cinnamaldehyde on cisplatin-induced nephrotoxicity in rats. J Biochem Mol Toxicol 27: 508-514, 2013.

20. Conti BJ, Búfalo MC, Golim Mde A, Bankova V and Sforcin JM: Cinnamic Acid is partially involved in propolis immunomodulatory action on human monocytes. Evid Based Complement Alternat Med 2013: 109864, 2013.

21. Adisakwattana S, Sompong W, Meeprom A, Ngamukote S and Yibchok-Anun S: Cinnamic acid and its derivatives inhibit fructose-mediated protein glycation. Int J Mol Sci 13: 1778-1789, 2012.

22. Kasetti RB, Nabi SA, Swapna S and Apparao C: Cinnamic acid as one of the antidiabetic active principle(s) from the seeds of Syzygium alternifolium. Food Chem Toxicol 50: 1425-1431, 2012.

23. Chen YL, Huang ST, Sun FM, Chiang YL, Chiang CJ, Tsai CM and Weng CJ: Transformation of cinnamic acid from trans- to cis-form raises a notable bactericidal and synergistic activity against multiple-drug resistant Mycobacterium tuberculosis. Eur J Pharm Sci 43: 188-194, 2011.

24. Barros MP, Lemos M, Maistro EL, Leite MF, Sousa JP, Bastos JK and Andrade SF: Evaluation of antiulcer activity of the main phenolic acids found in Brazilian Green Propolis. J Ethnopharmacol 120: 372-377, 2008.

25. Kong YH, Jo YO, Cho CW, Son D, Park S, Rho J and Choi SY: Inhibitory effects of cinnamic acid on melanin biosynthesis in skin. Biol Pharm Bull 31: 946-948, 2008.

26. Niero EL and Machado-Santelli GM: Cinnamic acid induces apoptotic cell death and cytoskeleton disruption in human melanoma cells. J Exp Clin Cancer Res 32: 31, 2013.

27. Tsai CM, Sun FM, Chen YL, Hsu CL, Yen GC and Weng CJ: Molecular mechanism depressing PMA-induced invasive behaviors in human lung adenocarcinoma cells by cis- and trans-cinnamic acid. Eur J Pharm Sci 48: 494-501, 2013.

28. Wheatley NC, Andrews KT, Tran TL, Lucke AJ, Reid RC and Fairlie DP: Antimalarial histone deacetylase inhibitors containing cinnamate or NSAID components. Bioorg Med Chem Lett 20: 7080-7084, 2010 .

29. Halley F, Reinshagen J, Ellinger B, Wolf M, Niles AL, Evans NJ, Kirkland TA, Wagner JM, Jung M, Gribbon P and Gul S: A bioluminogenic HDAC activity assay: validation and screening. J Biomol Screen 16: 1227-1235, 2011.

30. Wang LT, Liou JP, Li YH, Liu YM, Pan SL, and Teng CM. A novel class I HDAC inhibitor, MPT0G030, induces cell apoptosis and differentiation in human colorectal cancer cells via HDAC1/PKC $\delta$ and E-cadherin. Oncotarget 5: 5651-5662, 2014. 